\title{
Observability of Boolean control networks: A unified approach based on finite automata
}

\author{
Kuize Zhang, Member, IEEE, Lijun Zhang
}

\begin{abstract}
The problem on how to determine the observability of Boolean control networks (BCNs) has been open for five years already. In this paper, we propose a unified approach to determine all the four types of observability of BCNs in the literature. We define the concept of weighted pair graphs for BCNs. In the sense of each observability, we use the so-called weighted pair graph to transform a $\mathrm{BCN}$ to a finite automaton, and then we use the automaton to determine observability. In particular, the two types of observability that rely on initial states and inputs in the literature are determined. Finally, we show that no pairs of the four types of observability are equivalent, which reveals the essence of nonlinearity of BCNs.
\end{abstract}

Index Terms-Boolean control network, observability, weighted pair graph, finite automaton, formal language, semi-tensor product of matrices

\section{INTRODUCTION}

In 2007, Akutsu et al. [5] propose the concept of controllability of Boolean control networks (BCNs), prove that determining the controllability of BCNs is NP-hard ${ }^{1}$, and point out that "One of the major goals of systems biology is to develop a control theory for complex biological systems". Since then, the study on control-theoretic problems in the areas of Boolean networks (initiated by Kauffman [3] in 1969 to describe genetic regulatory networks) and Boolean control networks (initiated in [2] in 2001) has drawn vast attention (cf. [6]-[14] etc.). Controllability and observability are basic control-theoretic problems. In 2009, Cheng et al. [6] construct a control-theoretic framework for BCNs by using a new tool, called the semi-tensor product (STP) of matrices proposed in [4] in 2001, and give equivalent conditions for controllability of BCNs and observability of controllable BCNs. Since then, to the best of our knowledge, how to determine this observability has been open. This type of observability means that every initial state can be determined by an input sequence. Later on,

K. Zhang is with College of Automation, Harbin Engineering University, Harbin, 150001, PR China (e-mail: zkz0017@163.com), and Institute of Systems Science, Chinese Academy of Sciences, Beijing 100190, PR China. L. Zhang is with School of Marine Science and Technology, Northwestern Polytechnical University, Xi'an, 710072, PR China (e-mail: zhanglj7385@nwpu.edu.cn) and College of Automation, Harbin Engineering University, Harbin, 150001, PR China.

The original version of this paper was presented at the 33rd Chinese Control Conference, July 28-30, 2014, Nanjing, China.

This work was supported by the Fundamental Research Funds for the Central Universities (HEUCFX41501), National Natural Science Foundation of China (No. 61573288), Program for New Century Excellent Talents in University of Ministry of Education of China and Basic Research Foundation of Northwestern Polytechnical University (No. JC201230).

Manuscript received xxxx xx, xxxx; revised xxxx xx, xxxx.

${ }^{1}$ That is, there exists no polynomial time algorithm for determining the controllability of BCNs unless $\mathbf{P}=\mathbf{N P}$. important results on other types of observability of BCNs came up. Until now, there are four types of observability. Another observability, proposed in [7] in 2010, stands for that for every two distinct initial states, there exists an input sequence which can distinguish them. There is a sufficient but not necessary condition in [7]. However, there is no equivalent condition in [7]. This observability is determined in [16] in 2015 based on an algebraic method. A third observability stating that there is an input sequence that determines the initial state, is proposed in [8] to study the identifiability problem of BCNs in 2011. It is proved that determining this observability is NP-hard in [12] in 2013. Nevertheless, one way is proposed in [11] to determine this observability in 2013. A fourth observability is determined in [14], [15] ${ }^{2}$ in 2013, which is essentially the observability of linear control systems, i.e., every sufficiently long input sequence can determine the initial state.

Like nonlinear systems, BCNs are polynomial systems over $\mathbb{F}_{2}$, the Galois field of two elements [16]. This explains why the observability proposed in [6], [7] that rely on initial states and inputs are important for BCNs. The methods of determining the last two types of observability are not suitable for the first two, mainly because they are based on the independence of initial states and/or inputs. Besides, it is not known whether the method for the second type used in [16] is suitable for the other three types now. In this paper, we propose a unified method based on finite automata to determine all the four types of observability regardless of dependence. To this end, we firstly define weighted pair graphs for BCNs, which consist of pairs of states of BCNs producing the same outputs, and transitions between the pairs. Secondly, we use the weighed pair graph to transform a $\mathrm{BCN}$ to a deterministic finite automaton. Finally, we use the automaton to determine observability.

The remainder of this paper is organized as follows. Section II introduces necessary preliminaries about STP, BCNs with their algebraic forms, formal languages and finite automata. Section III presents the algorithms to determine all the four types of observability. Section IV shows the pairwise nonequivalence of the four types of observability of BCNs. Section V ends up with some remarks and challenging open problems.

\section{Preliminaries}

\section{A. The semi-tensor product of matrices}

We first introduce some related notations in STP.

${ }^{2}$ Note that the types of observability studied in [14], [15] are the same. 
- $2^{A}$ : the power set of set $A$

- $\mathbb{Z}_{+}$: the set of positive integers

- $\mathbb{N}$ : the set of natural numbers

- $\mathcal{D}$ : the set $\{0,1\}$

- $\delta_{n}^{i}$ : the $i$-th column of the identity matrix $I_{n}$

- $\Delta_{n}$ : the set $\left\{\delta_{n}^{1}, \ldots, \delta_{n}^{n}\right\}\left(\Delta:=\Delta_{2}\right)$

- $\delta_{n}\left[i_{1}, \ldots, i_{s}\right]:$ the logical matrix $\left[\delta_{n}^{i_{1}}, \ldots, \delta_{n}^{i_{s}}\right]$ $\left(i_{1}, \ldots, i_{s} \in\{1,2, \ldots, n\}\right.$ ) (for the concept of logical matrices, we refer the reader to [9].)

- $\mathcal{L}_{n \times s}$ : the set of $n \times s$ logical matrices, i.e., $\left\{\delta_{n}\left[i_{1}, \ldots, i_{s}\right] \mid i_{1}, \ldots, i_{s} \in\{1,2, \ldots, n\}\right\}$

- $[1, N]$ : the first $N$ positive integers

- $|A|:$ the cardinality of set $A$

Definition 1: [9] Let $A \in \mathbb{R}_{m \times n}, B \in \mathbb{R}_{p \times q}$, and $\alpha=$ $\operatorname{lcm}(n, p)$ be the least common multiple of $n$ and $p$. The STP of $A$ and $B$ is defined as $A \ltimes B=\left(A \otimes I_{\frac{\alpha}{n}}\right)\left(B \otimes I_{\frac{\alpha}{p}}\right)$, where $\otimes$ denotes the Kronecker product.

From this definition, it is easy to see that the conventional product of matrices is a particular case of STP. Since STP keeps most properties of the conventional product [9], e.g., the associative law, the distributive law, etc., we usually omit the symbol " $\ltimes$ " hereinafter.

\section{B. Boolean control networks and their algebraic forms}

In this paper, we investigate the following $\mathrm{BCN}$ with $n$ state nodes, $m$ input nodes and $q$ output nodes:

$$
\begin{aligned}
& x(t+1)=f(u(t), x(t)), \\
& y(t)=h(x(t)),
\end{aligned}
$$

where $x \in \mathcal{D}^{n} ; u \in \mathcal{D}^{m} ; y \in \mathcal{D}^{q} ; t=0,1, \ldots ; f: \mathcal{D}^{n+m} \rightarrow$ $\mathcal{D}^{n}$ and $h: \mathcal{D}^{n} \rightarrow \mathcal{D}^{q}$ are logical functions.

Using the STP of matrices, (1) can be equivalently represented in the following algebraic form [6]

$$
\begin{aligned}
& x(t+1)=L u(t) x(t), \\
& y(t)=H x(t),
\end{aligned}
$$

where $x \in \Delta_{N}, u \in \Delta_{M}$ and $y \in \Delta_{Q}$ denote states, inputs and outputs, respectively; $t=0,1, \ldots ; L \in \mathcal{L}_{N \times(N M)} ; H \in$ $\mathcal{L}_{Q \times N}$; hereinafter, $N:=2^{n}, M:=2^{m}$ and $Q:=2^{q}$.

For more details on properties of STP, and how to transform a $\mathrm{BCN}$ into its equivalent algebraic form, we refer the reader to $[6]$.

\section{Formal languages and finite automata}

The theories of formal languages and finite automata are among the mathematical foundations of theoretical computer science [1]. Let $\Sigma$ be a finite nonempty set (called alphabet). We use $\Sigma^{*}$ to denote the set of all finite sequences (called words) of elements (called letters) of $\Sigma$. The empty word is denoted by $\epsilon .|u|$ denotes the length of word $u$. For example, $|a b c|=3$ for the alphabet $\{a, b, c\},|\epsilon|=0$. The set of all words of length $p$ is denoted by $\Sigma^{p}$. Notice that $\Sigma^{0}=\{\epsilon\}$. Then $\Sigma^{*}=\cup_{p=0}^{\infty} \Sigma^{p}$. A formal language (or language for short) is a subset of $\Sigma^{*}$.

A deterministic finite automaton (DFA) is defined as 5-tuple $A=\left(S, \Sigma, \sigma, s_{0}, F\right)$, where $S$ denotes the finite state set, $\Sigma$ the finite alphabet, $s_{0} \in S$ the initial state, $F \subset S$ the final state set, and $\sigma: S \times \Sigma \rightarrow S$ the transition partial function, i.e., a function defined on a fixed subset of $S \times \Sigma$, which can naturally be extended to $\sigma: S \times \Sigma^{*} \rightarrow S$. We call a DFA complete if $\sigma$ is a function from $S \times \Sigma^{*}$ to $S$. A language $L$ over alphabet $\Sigma$ is called regular, if it is recognized by a DFA $A=\left(S, \Sigma, \sigma, s_{0}, F\right)$, i.e., $L=\left\{w \in \Sigma^{*} \mid \sigma\left(s_{0}, w\right) \in F\right\}$. A word $u \in \Sigma^{*}$ such that $\sigma\left(s_{0}, u\right) \in F$ is called accepted by DFA $A$. A DFA accepts the empty word $\epsilon$ iff its initial state is final.

In order to represent a DFA, we introduce the transition graph of DFA $A=\left(S, \Sigma, \sigma, s_{0}, F\right)$. Let $V, E$ and $W$ be the vertex set, the edge set and the weight function of a weighted directed graph $G=(V, E, W)$. $G$ is called the transition graph of DFA $A$, if $V=S, E=\left\{\left(s_{i}, s_{j}\right) \in V \times V \mid\right.$ there is $a \in$ $\Sigma$ such that $\left.\sigma\left(s_{i}, a\right)=s_{j}\right\} \subset V \times V$, and $W: E \rightarrow 2^{\Sigma}$, $\left(s_{i}, s_{j}\right) \mapsto\left\{a \in \Sigma \mid \sigma\left(s_{i}, a\right)=s_{j}\right\}$.

In the transition graph of a DFA, we add a "start" input arrow to the vertex of the initial state, and use double circles to denote final states. We omit the curly bracket " \{\} " in the weights of edges. See Fig. 3 for an example.

Now we give a proposition on finite automata that will be used in the main results.

Proposition 2.1: Given a DFA $A=\left(S, \Sigma, \sigma, s_{0}, F\right)$. Assume that $F=S$ and for each $s \in S$, there is a word $u \in \Sigma^{*}$ such that $\sigma\left(s_{0}, u\right)=s$. Then $L(A)=\Sigma^{*}$ iff $A$ is complete.

Proof: "if": If $A$ is complete and $F=S$, then $\epsilon \in L(A)$ and for any nonempty word $w \in \Sigma^{*}, \sigma\left(s_{0}, w\right) \in F$, i.e., $w \in L(A)$. Hence $L(A)=\Sigma^{*}$.

"only if": Assume that $F=S$ and $A$ is not complete. Choose an $s \in S$ such that $\sigma$ is not well defined at $(s, a)$ for some $a \in \Sigma$. Choose word $w \in \Sigma^{*}$ such that $\sigma\left(s_{0}, w\right)=s$, then word $w a \notin L(A)$, for $A$ is deterministic. That is, $L(A) \neq$ $\Sigma^{*}$.

\section{DETERMINING THE OBSERVABILITY OF BCNS}

\section{A. Weighted pair graph}

In this subsection, we define a weighted directed graph for BCN (2), named weighted pair graph. Based on the weighted pair graph, in the following subsections, we construct a DFA in the sense of each observability, and then use the obtained DFA and Proposition 2.1 to determine observability.

Definition 2: Consider BCN (2). Let $\mathcal{V}, \mathcal{E}$ and $\mathcal{W}$ be the vertex set, the edge set and the weight function of a weighted directed graph $\mathcal{G}=(\mathcal{V}, \mathcal{E}, \mathcal{W})$. $\mathcal{G}$ is called the weighted pair graph of the BCN, if $\mathcal{V}=\left\{\left(x, x^{\prime}\right) \in \Delta_{N} \times \Delta_{N} \mid H x=\right.$ $\left.H x^{\prime}\right\}^{3}, \mathcal{E}=\left\{\left(\left(x_{1}, x_{1}^{\prime}\right),\left(x_{2}, x_{2}^{\prime}\right)\right) \in \mathcal{V} \times \mathcal{V} \mid\right.$ there exists $u \in$ $\Delta_{M}$ such that $\operatorname{Lux}_{1}=x_{2}$ and $\operatorname{Lux}_{1}^{\prime}=x_{2}^{\prime}$, or, $\operatorname{Lu} x_{1}=$ $x_{2}^{\prime}$ and $\left.L u x_{1}^{\prime}=x_{2}\right\} \subset \mathcal{V} \times \mathcal{V}$, and $\mathcal{W}: \mathcal{E} \rightarrow 2^{\Delta_{M}}$, $\left(\left(x_{1}, x_{1}^{\prime}\right),\left(x_{2}, x_{2}^{\prime}\right)\right) \mapsto\left\{u \in \Delta_{M} \mid L u_{1} x_{1}=x_{2}\right.$ and $L u_{1} x_{1}^{\prime}=$ $x_{2}^{\prime}$, or, $L u x_{1}=x_{2}^{\prime}$ and $\left.L u x_{1}^{\prime}=x_{2}\right\}$.

Intuitively, there is an edge from a vertex $v$ to another one $v^{\prime}$, iff there is an input $u$ driving one state in $v$ to one state in $v^{\prime}$ and driving the other state in $v$ to the other state in $v^{\prime}$. Similar to the transition graph of a DFA, we omit the curly

\footnotetext{
${ }^{3}$ Here $\left(x, x^{\prime}\right)$ is an unordered pair, i.e., $\left(x, x^{\prime}\right)=\left(x^{\prime}, x\right)$.
} 


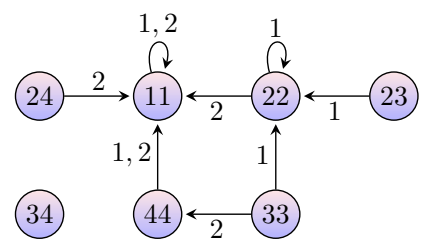

Fig. 1. The weighted pair graph of BCN (3), where the number $i j$ in each circle denotes the state pair $\left(\delta_{4}^{i}, \delta_{4}^{j}\right)$, and the weight $k_{1}, k_{2}, \ldots$ beside each edge denotes the weight $\left\{\delta_{2}^{k_{1}}, \delta_{2}^{k_{2}}, \ldots\right\}$ of the edge.

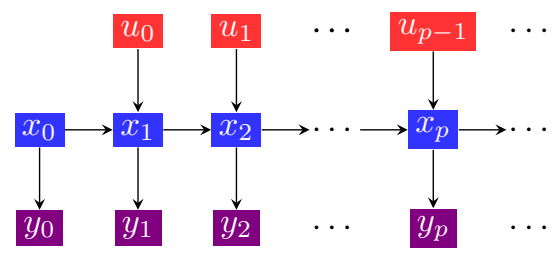

Fig. 2. The input-state-output-time transfer graph of BCN (2), where subscripts stand for time steps, $x_{0}, x_{1}, \ldots$ states, $u_{0}, u_{1}, \ldots$ inputs, $y_{1}, y_{2}, \ldots$ outputs, and arrows infer dependence.

bracket " \{\} " in the weights of edges. Hereinafter, we call each vertex $(x, x) \in \Delta_{N} \times \Delta_{N}$ a diagonal vertex.

From Definition 2, the weighted pair graph consists of every state pair producing the same output. In fact, to test whether a $\mathrm{BCN}$ is observable, is to test whether these states can be distinguished by input sequences.

Let $(\mathcal{V}, \mathcal{E}, \mathcal{W})$ be a weighted pair graph. For a subset $V$ of $\mathcal{V}$, the subgraph generated by $V$ is the graph $(V, \mathcal{E} \cap(V \times$ $\left.V),\left.\mathcal{W}\right|_{\mathcal{E} \cap(V \times V)}\right)$, where $\left.\mathcal{W}\right|_{\mathcal{E} \cap(V \times V)}$ is the restriction of $\mathcal{W}$ to $\mathcal{E} \cap(V \times V)$.

The weighted pair graph of the following $\mathrm{BCN}$ (3) is depicted in Fig. 1.

$$
\begin{aligned}
x(t+1) & =\delta_{4}[1,1,2,1,2,4,1,1] x(t) u(t), \\
y(t) & =\delta_{2}[1,2,2,2] x(t),
\end{aligned}
$$

where $t \in \mathbb{N}, x \in \Delta_{4}, y, u \in \Delta$.

\section{B. Notations}

The input-state-output-time transfer graph of BCN (2) is drawn in Fig. 2. In order to define these observability, we define the following mappings:

Let $\Delta_{M}, \Delta_{N}, \Delta_{Q}$ be three alphabets. For all $x_{0} \in \Delta_{N}$ and all $p \in \mathbb{Z}_{+}$,

1)

$$
\begin{aligned}
& L_{x_{0}}^{p}:\left(\Delta_{M}\right)^{p} \rightarrow\left(\Delta_{N}\right)^{p}, u_{0} \ldots u_{p-1} \mapsto x_{1} \ldots x_{p}, \\
& L_{x_{0}}^{\mathbb{N}}:\left(\Delta_{M}\right)^{\mathbb{N}} \rightarrow\left(\Delta_{N}\right)^{\mathbb{N}}, u_{0} u_{1} \ldots \mapsto x_{1} x_{2} \ldots
\end{aligned}
$$

2)

$$
\begin{aligned}
& (H L)_{x_{0}}^{p}:\left(\Delta_{M}\right)^{p} \rightarrow\left(\Delta_{Q}\right)^{p}, u_{0} \ldots u_{p-1} \mapsto y_{1} \ldots y_{p}, \\
& (H L)_{x_{0}}^{\mathbb{N}}:\left(\Delta_{M}\right)^{\mathbb{N}} \rightarrow\left(\Delta_{Q}\right)^{\mathbb{N}}, u_{0} u_{1} \ldots \mapsto y_{1} y_{2} \ldots
\end{aligned}
$$

For all $p \in \mathbb{Z}_{+}$, all $U=u_{1} \ldots u_{p} \in\left(\Delta_{M}\right)^{p}$, and all $1 \leq$ $i \leq j \leq|U|$, we use $U[i, j]$ to denote the word $u_{i} \ldots u_{j}$. In particular, $U[i]$ (or $U(i)$ ) is short for $U[i, i]$. Given $U \in$
$\left(\Delta_{M}\right)^{*}, U^{\infty}$ denotes the concatenation of infinite copies of $U$, i.e., $U U \ldots$ For all input sequences $U=u_{0} u_{1} \ldots \in\left(\Delta_{M}\right)^{\mathbb{N}}$, and all $0 \leq i \leq j \in \mathbb{N}$, we use $U[i, j]$ to denote the word $u_{i} \ldots u_{j}$.

\section{Determining the observability in [6]}

Definition 3 ( [6]): BCN (2) is called observable, if for every initial state $x_{0} \in \Delta_{N}$, there exists an input sequence such that the initial state can be determined by the output sequence.

Definition 3 can be expressed equivalently as follows:

Definition 4: BCN (2) is called observable, if for every initial state $x_{0} \in \Delta_{N}$, there exists an input sequence $U \in\left(\Delta_{M}\right)^{p}$ for some $p \in \mathbb{Z}_{+}$such that for all states $x_{0} \neq \bar{x}_{0} \in \Delta_{N}$, $H x_{0}=H \bar{x}_{0}$ implies $(H L)_{x_{0}}^{p}(U) \neq(H L)_{\bar{x}_{0}}^{p}(U)$.

In this subsection, the observability of $\mathrm{BCN}$ (2) refers to Definition 4.

According to Definition 4, BCN (2) is not observable iff there is a state $\delta_{N}^{i}$ in a non-diagonal vertex of its weighted pair graph $\mathcal{G}=(\mathcal{V}, \mathcal{E}, \mathcal{W})$ such that for all $p \in \mathbb{Z}_{+}$, all $U \in$ $\left(\Delta_{M}\right)^{p}$, there is a state $\delta_{N}^{j}$ with $j \neq i,\left(\delta_{N}^{i}, \delta_{N}^{j}\right) \in \mathcal{V}$ and $(H L)_{\delta_{N}^{i}}^{p}(U)=(H L)_{\delta_{N}^{j}}^{p}(U)$

For fixed $\delta_{N}^{i}$, we design an algorithm to construct a DFA for BCN (2) according to its weighted pair graph $(\mathcal{V}, \mathcal{E}, \mathcal{W})$. The new DFA is denoted by $A_{\delta_{N}^{i}}$, and accepts exactly all finite input sequences that do not determine $\delta_{N}^{i}$. The states of DFA $A_{\delta_{N}^{i}}$ are subsets of $\mathcal{V}$.

Algorithm 3.1: 1) Set $\Delta_{M}$ to be the alphabet of the DFA.

Set the subset of $\mathcal{V}$ consisting of all the non-diagonal vertices of $\mathcal{V}$ that contain $\delta_{N}^{i}$ to be the initial state of the DFA. That is, the set $s_{0}:=\left\{\left(\delta_{N}^{k}, \delta_{N}^{l}\right) \mid k, l \in\right.$ $[1, N], H \delta_{N}^{k}=H \delta_{N}^{l}, k \neq l, k$ or $\left.l=i\right\}$ is the initial state of the DFA.

2) For each letter $\delta_{M}^{j}, j \in[1, M]$, find the value for the transition partial function of the DFA at $\left(s_{0}, \delta_{M}^{j}\right)$. The specific procedure is as follows:

Fix $j \in[1, M]$. Set $s_{j}:=\left\{v \in \mathcal{V} \mid\right.$ there is $v^{\prime} \in$ $s_{0}$ such that $\left(v^{\prime}, v\right) \in \mathcal{E}$, and $\left.\delta_{M}^{j} \in \mathcal{W}\left(\left(v^{\prime}, v\right)\right)\right\}$. If $s_{j} \neq \emptyset$, add $s_{j}$ to the state set of the DFA and set $s_{j}$ to be the value of the transition partial function at $\left(s_{0}, \delta_{M}^{j}\right)$; otherise, the transition partial function is not well defined at $\left(s_{0}, \delta_{M}^{j}\right)$.

3) For each new state $s$ of the DFA found in the previous step, and each letter $\delta_{M}^{j}, j \in[1, M]$, find the value for the transition partial function at $\left(s, \delta_{M}^{j}\right)$ according to Step 2 .

4) Repeat Step 3 until no new state of the DFA occurs. (Since $\mathcal{V}$ is a finite set, so is its power set, this repetition will stop.)

5) Set all the states of the DFA to be final states.

Take BCN (3) for example. Choose state $\delta_{4}^{2}$. Then the DFA $A_{\delta_{4}^{2}}$ generated by Algorithm 3.1 is as shown in Fig. 3.

Now we give a necessary and sufficient condition for this observability.

Theorem 3.2: BCN (2) is not observable in the sense of Definition 4 iff there is a state $\delta_{N}^{i}$ in a non-diagonal vertex of its weighted pair graph such that the DFA $A_{\delta_{N}^{i}}$ generated by Algorithm 3.1 recognizes language $\left(\Delta_{M}\right)^{*}$. 


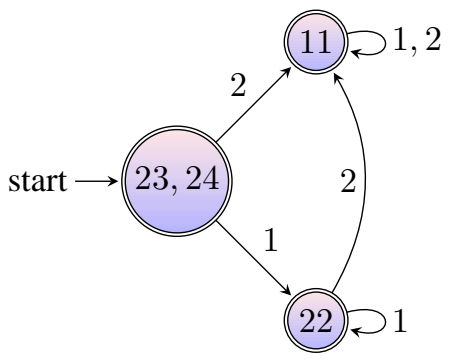

Fig. 3. The DFA $A_{\delta_{4}^{2}}$ with respect to BCN (3) generated by Algorithm 3.1, where the number $i j$ in each circle denotes the state pair $\left(\delta_{4}^{i}, \delta_{4}^{j}\right)$, and the weight $k$ beside each edge denotes the input $\delta_{2}^{k}$.

Proof: "only if": Assume that BCN (2) is not observable, then there is a state $\delta_{N}^{i}$ such that for all $p \in \mathbb{Z}_{+}$, all $U \in$ $\left(\Delta_{M}\right)^{p}$, there is a state $\delta_{N}^{j}$ satisfying $i \neq j, H \delta_{N}^{i}=H \delta_{N}^{j}$, and $(H L)_{\delta_{N}^{i}}^{p}(U)=(H L)_{\delta_{N}^{j}}^{p}(U)$. According to Algorithm 3.1, $v_{0}:=\left(\delta_{N}^{i}, \delta_{N}^{j}\right)$ is in the initial state of DFA $A_{\delta_{N}^{i}}$. Denote the weighted pair graph of BCN (2) by $\mathcal{G}=(\mathcal{V}, \mathcal{E}, \mathcal{W})$. Then there exist vertices $v_{k}:=\left(\delta_{N}^{i_{k}}, \delta_{N}^{j_{k}}\right) \in \mathcal{V}$ such that $U[k] \in$ $\mathcal{W}\left(\left(v_{k-1}, v_{k}\right)\right), k=1, \ldots, p$. That is, for all $p \in \mathbb{Z}_{+}$, each $U$ in $\left(\Delta_{M}\right)^{p}$ is accepted by DFA $A_{\delta_{N}^{i}}$. It is obvious that $\epsilon \in$ $L\left(A_{\delta_{N}^{i}}\right)$. Then $L\left(A_{\delta_{N}^{i}}\right)=\left(\Delta_{M}\right)^{*}$.

"if": Note that the DFA $A_{\delta_{N}^{i}}$ accepts exactly all finite input sequences that do not determine $\delta_{N}^{i}$. Then $L\left(A_{\delta_{N}^{i}}\right)=\left(\Delta_{M}\right)^{*}$ implies that for all $p \in \mathbb{Z}_{+}$, all $U \in\left(\Delta_{M}\right)^{p}$, there is a state $\delta_{N}^{j}$ such that $i \neq j, H \delta_{N}^{i}=H \delta_{N}^{j}$, and $(H L)_{\delta_{N}^{i}}^{p}(U)=$ $(H L)_{\delta_{N}^{j}}^{p}(U)$. That is, BCN (2) is not observable.

Proposition 2.1, Theorem 3.2 and Algorithm 3.1 directly imply the following result that can be used to check whether a given $\mathrm{BCN}$ is observable.

Theorem 3.3: BCN (2) is not observable in the sense of Definition 4 iff there is a state $\delta_{N}^{i}$ in a non-diagonal vertex of its weighted pair graph such that the DFA $A_{\delta_{N}^{i}}$ generated by Algorithm 3.1 is complete.

Example 3.4: Check whether BCN (3) is observable.

According to Theorem 3.3, we should check $\delta_{4}^{2}, \delta_{4}^{3}, \delta_{4}^{4}$ one by one.

First we check $\delta_{4}^{2}$. According to Algorithm 3.1, we calculate DFA $A_{\delta_{4}^{2}}$, and derive the transition graph of this DFA as shown in Fig. 3. This DFA is complete, by Theorem 3.3, BCN (3) is not observable.

\section{Determining the observability in [7]}

Definition 5: BCN (2) is called observable, if for any distinct states $x_{0}, \bar{x}_{0} \in \Delta_{N}$, there is an input sequence $U \in\left(\Delta_{M}\right)^{p}$ for some $p \in \mathbb{Z}_{+}$, such that $H x_{0}=H \bar{x}_{0}$ implies $(H L)_{x_{0}}^{p}(U) \neq(H L)_{\bar{x}_{0}}^{p}(U){ }^{4}$

In this subsection, the observability of BCN (2) means Definition 5.

According to Definition 5, BCN (2) is not observable iff there is a non-diagonal vertex $\left(\delta_{N}^{i}, \delta_{N}^{j}\right)$ in its weighted

\footnotetext{
${ }^{4}$ Actually, after removing " $H x_{0}=H \bar{x}_{0}$ implies" in Definition 5, Definition 5 becomes the observability studied in [7]. In order to make the observability studied in [7] exactly the widely accepted one for nonlinear control systems, we modify it in Definition 5.
}

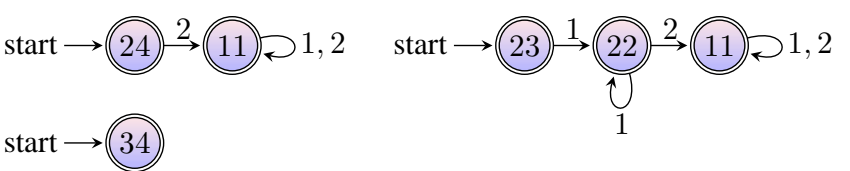

Fig. 4. The DFA of each non-diagonal vertex of the weighted pair graph of BCN (3) generated by Algorithm 3.5, where the number $i j$ in each circle denotes the state pair $\left(\delta_{4}^{i}, \delta_{4}^{j}\right)$, and the weight $k$ beside each edge denotes the input $\delta_{2}^{k}$.

pair graph such that for all $p \in \mathbb{Z}_{+}$, and $U \in\left(\Delta_{M}\right)^{p}$, $(H L)_{\delta_{N}^{i}}^{p}(U)=(H L)_{\delta_{N}^{j}}^{p}(U)$.

For a fixed non-diagonal vertex $\left(\delta_{N}^{i}, \delta_{N}^{j}\right)$, we design an algorithm to construct a DFA for BCN (2) according to its weighted pair graph. The new DFA is denoted by $A_{\left(\delta_{N}^{i}, \delta_{N}^{j}\right)}$, and accepts exactly all finite input sequences that do not distinguish $\delta_{N}^{i}$ and $\delta_{N}^{j}$.

Algorithm 3.5: 1) Set $\Delta_{M}$ to be the alphabet of the DFA. Set vertex $\left(\delta_{N}^{i}, \delta_{N}^{j}\right)$ to be the initial state of the DFA.

2) Find each vertex $v$ such that there is a path from $\left(\delta_{N}^{i}, \delta_{N}^{j}\right)$ to $v$. Keep the subgraph generated by $\left(\delta_{N}^{i}, \delta_{N}^{j}\right)$ and those vertices, and remove all vertices and edges outside of the subgraph.

3) Set each remainder vertex to be a final state of the DFA.

Again take BCN (3) as an example. The DFA of each non-diagonal vertex of the weighted pair graph generated by Algorithm 3.5 is shown in Fig. 4.

The following is a necessary and sufficient condition for this observability.

Theorem 3.6: BCN (2) is not observable in the sense of Definition 5 iff there is a non-diagonal vertex $\left(\delta_{N}^{i}, \delta_{N}^{j}\right)$ in its weighted pair graph such that the DFA $A_{\left(\delta_{N}^{i}, \delta_{N}^{j}\right)}$ generated by Algorithm 3.5 recognizes language $\left(\Delta_{M}\right)^{*}$.

Proof: "only if": Assume that BCN (2) is not observable, then there is a non-diagonal vertex $\left(\delta_{N}^{i}, \delta_{N}^{j}\right)$ in the weighted pair graph of $\mathrm{BCN}$ (2) such that for all $p \in \mathbb{Z}_{+}$, all $U \in$ $\left(\Delta_{M}\right)^{p},(H L)_{\delta_{N}^{i}}^{p}(U)=(H L)_{\delta_{N}^{j}}^{p}(U)$. Then for all $p \in \mathbb{Z}_{+}$, each $U$ in $\left(\Delta_{M}\right)^{p}$ is accepted by DFA $A_{\left(\delta_{N}^{i}, \delta_{N}^{j}\right)}$. It is obvious that $\epsilon \in L\left(A_{\left(\delta_{N}^{i}, \delta_{N}^{j}\right)}\right)$. Then $L\left(A_{\left(\delta_{N}^{i}, \delta_{N}^{j}\right)}\right)=\left(\Delta_{M}\right)^{*}$.

"if": Obvious by Definition 5.

From Proposition 2.1, Theorem 3.6 and Algorithm 3.5, the following result which follows can be used to determine whether a given $\mathrm{BCN}$ is observable.

Theorem 3.7: $\mathrm{BCN}$ (2) is not observable in the sense of Definition 5 iff there is a non-diagonal vertex $\left(\delta_{N}^{i}, \delta_{N}^{j}\right)$ in its weighted pair graph such that the DFA $A_{\left(\delta_{N}^{i}, \delta_{N}^{j}\right)}$ generated by Algorithm 3.5 is complete.

Example 3.8: Check whether $\mathrm{BCN}$ (3) is observable. According to Theorem 3.7, one should check $\left(\delta_{4}^{2}, \delta_{4}^{3}\right),\left(\delta_{4}^{2}, \delta_{4}^{4}\right),\left(\delta_{4}^{3}, \delta_{4}^{4}\right)$ one by one. From Fig. 4, one sees that $\delta_{2}^{2} \notin L\left(A_{\left(\delta_{4}^{2}, \delta_{4}^{3}\right)}\right), \quad \delta_{2}^{1} \notin L\left(A_{\left(\delta_{4}^{2}, \delta_{4}^{4}\right)}\right)$, and $\delta_{2}^{1}, \delta_{2}^{2} \notin L\left(A_{\left(\delta_{4}^{3}, \delta_{4}^{4}\right)}\right)$. Then by Theorem 3.7, BCN (3) is observable.

At the end of this subsection, using the concept of weighted pair graphs, we give a further result on this observability.

Theorem 3.9: Consider BCN (2). Denote the number of non-diagonal vertices of its weighted pair graph by $N_{n d}$. The 
following two items are equivalent.

(i) The $\mathrm{BCN}$ is observable in the sense of Definition 5.

(ii) $N_{n d}=0$ or for all distinct states $x_{0}, \bar{x}_{0} \in \Delta_{N}$, there is an input sequence $U \in\left(\Delta_{M}\right)^{N_{n d}}$ such that $H x_{0}=H \bar{x}_{0}$ implies $(H L)_{x_{0}}^{N_{n d}}(U) \neq(H L)_{\bar{x}_{0}}^{N_{n d}}(U)$.

Proof: (ii) $\Rightarrow$ (i):

Obvious by Definition 5 .

(i) $\Rightarrow$ (ii):

Assume that (ii) does not hold. That is, $N_{n d}>0$ and there are distinct $x, x^{\prime} \in \Delta_{N}$, for all $U \in\left(\Delta_{M}\right)^{N_{n d}}$, $H x=H x^{\prime}$ and $(H L)_{x}^{N_{n d}}(U)=(H L)_{x^{\prime}}^{N_{n d}}(U)$. Use Algorithm 3.5 to generate DFA $A_{\left(x, x^{\prime}\right)}=\left(S, \Delta_{M}, \sigma,\left(x, x^{\prime}\right), S\right)$. Then $\cup_{i=0}^{N_{n d}}\left(\Delta_{M}\right)^{i} \subset L\left(A_{\left(x, x^{\prime}\right)}\right)$. We claim that $A_{\left(x, x^{\prime}\right)}$ is complete. Suppose the contrary: there is a state $v$ of $A_{\left(x, x^{\prime}\right)}$ and an input $u \in \Delta_{M}$ such that $\sigma$ is not well defined at $(v, u)$. Then $v$ is a non-diagonal vertex of the weighed pair graph, because for all diagonal vertices $v^{\prime}$ (if exist), for all inputs $u^{\prime} \in \Delta_{M}, \sigma$ is well defined at $\left(v^{\prime}, u^{\prime}\right)$. There are exactly $N_{n d}$ non-diagonal vertices, then there exists an input sequence $U_{1}$ of length less than $N_{n d}$ such that $\sigma\left(\left(x, x^{\prime}\right), U_{1}\right)=v$. We get a contradiction $U_{1} u \in \cup_{i=0}^{N_{n d}}\left(\Delta_{M}\right)^{i} \backslash L\left(A_{\left(x, x^{\prime}\right)}\right)$. By Theorem 3.7, the $\mathrm{BCN}$ is not observable.

\section{E. Determining the observability in [8]}

Definition 6 ( [8]): $\mathrm{BCN}$ (2) is called observable, if there exists an input sequence $U \in\left(\Delta_{M}\right)^{p}$ for some $p \in \mathbb{Z}_{+}$, such that for any distinct states $x_{0}, \bar{x}_{0} \in \Delta_{N}, H x_{0}=H \bar{x}_{0}$ implies $(H L)_{x_{0}}^{p}(U) \neq(H L)_{\bar{x}_{0}}^{p}(U)$.

In this subsection, the observability of BCN (2) refers to Definition 6.

According to Definition 6, to judge whether BCN (2) is observable, we need to check the set $\mathcal{V}_{n}$ of all non-diagonal vertices of its weighted pair graph $(\mathcal{V}, \mathcal{E}, \mathcal{W})$.

Now we design an algorithm to construct a DFA for BCN (2) according to its weighted pair graph $(\mathcal{V}, \mathcal{E}, \mathcal{W})$. The new DFA is denoted by $A_{\mathcal{V}_{n}}$, and accepts exactly every finite input sequence by which not all non-diagonal state pairs can be distinguished. The states of the DFA $A_{\mathcal{V}_{n}}$ are subsets of $\mathcal{V}$.

Algorithm 3.10: 1) Set $\Delta_{M}$ to be the alphabet of the DFA. Set the set $\mathcal{V}_{n}$ of all non-diagonal vertices of $\mathcal{V}$ to be the initial state of the DFA.

2) For each letter $\delta_{M}^{j}, j \in[1, M]$, find the value for the transition partial function of the DFA at $\left(\mathcal{V}_{n}, \delta_{M}^{j}\right)$. The specific procedure is as follows:

For each $j \in[1, M]$, let $s_{j}:=\left\{v \in \mathcal{V} \mid\right.$ there is $v^{\prime} \in$ $\mathcal{V}_{n}$ such that $\left(v^{\prime}, v\right) \in \mathcal{E}$, and $\left.\delta_{M}^{j} \in \mathcal{W}\left(\left(v^{\prime}, v\right)\right)\right\}$. If $s_{j} \neq \emptyset$, add $s_{j}$ to the state set of the DFA and set $s_{j}$ to be the value of the transition partial function at $\left(\mathcal{V}_{n}, \delta_{M}^{j}\right)$; otherwise, the transition partial function of the DFA is not well defined at $\left(\mathcal{V}_{n}, \delta_{M}^{j}\right)$.

3) For each new state $s$ of the DFA found in the previous step, for each letter $\delta_{M}^{j}, j \in[1, M]$, find the value for the transition partial function of the DFA at $\left(s, \delta_{M}^{j}\right)$ according to Step 2.

4) Repeat Step 3 until no new state of the DFA occurs. (Since $\mathcal{V}$ is a finite set, so is its power set, this repetition will stop.)

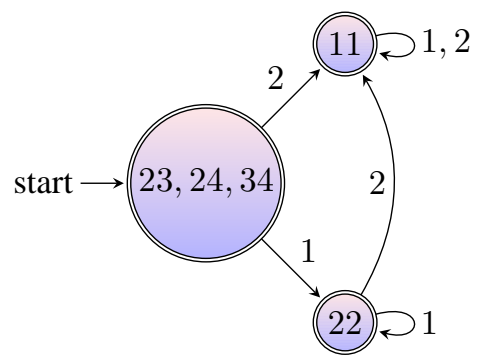

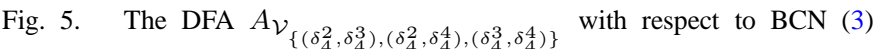
generated by Algorithm 3.10, where the number $i j$ in each circle denotes the state pair $\left(\delta_{4}^{i}, \delta_{4}^{j}\right)$, and the weight $k$ beside each edge denotes the input $\delta_{2}^{k}$.

5) Set all the states of the obtained DFA to be final states.

According to Algorithm 3.10, the following theorem holds.

Theorem 3.11: $\mathrm{BCN}$ (2) is not observable in the sense of Definition 6 iff the DFA $A_{\mathcal{V}_{n}}$ generated by Algorithm 3.10 recognizes language $\left(\Delta_{M}\right)^{*}$.

Proof: Notice that BCN (2) is not observable iff none of finite input sequences can distinguish all state pairs of $\mathcal{V}_{n}$, that is, $L\left(A_{\mathcal{V}_{n}}\right)=\left(\Delta_{M}\right)^{*}$.

From Proposition 2.1, Theorem 3.11 and Algorithm 3.10, the following result which follows can be used to judge whether BCN (2) is observable.

Theorem 3.12: BCN (2) is not observable in the sense of Definition 6 iff the DFA $A_{\mathcal{V}_{n}}$ generated by Algorithm 3.10 is complete.

Example 3.13: Check whether BCN (3) is observable.

According to Theorem 3.12, we should check whether DFA $A_{\left\{\left(\delta_{4}^{2}, \delta_{4}^{3}\right),\left(\delta_{4}^{2}, \delta_{4}^{4}\right),\left(\delta_{4}^{3}, \delta_{4}^{4}\right)\right\}}$ is complete.

From Fig. 5, one sees that this DFA is complete. Then by Theorem 3.12, BCN (3) is not observable.

Remark 3.1: In [12], it is proved that determining this observability is NP-hard. Actually, the results of [12] show that determining each of the four types of observability is NPhard. How to determine this observability has been solved in [11] by enumerating all possible input sequences of a common finite length. However, one can use our method to find any input sequence that determines the initial state. Due to the independence of initial states, their method cannot be applied to deal with Definitions 4 or 5.

\section{F. Determining the observability in [14]}

Definition 7 ( [14]): BCN (2) is called observable, if for any distinct states $x_{0}, \bar{x}_{0} \in \Delta_{N}$, for any input sequence $U \in$ $\left(\Delta_{M}\right)^{\mathbb{N}}, H x_{0}=H \bar{x}_{0}$ implies $(H L)_{x_{0}}^{\mathbb{N}}(U) \neq(H L)_{\bar{x}_{0}}^{\mathbb{N}}(U)$.

In this subsection, the observability of $\mathrm{BCN}$ (2) means Definition 7.

According to Definition 7, BCN (2) is not observable iff there are two distinct states $\delta_{N}^{i}, \delta_{N}^{j}$ and an input sequence $U \in\left(\Delta_{M}\right)^{\mathbb{N}}$ such that $H \delta_{N}^{i}=H \delta_{N}^{j}$ and $(H L)_{\delta_{N}^{i}}^{\mathbb{N}}(U)=$ $(H L)_{\delta_{N}^{j}}^{\mathbb{N}}(U)$. Then the following theorem can be used to determine this observability.

Theorem 3.14: BCN (2) is not observable in the sense of Definition 7 iff there is a non-diagonal vertex $\left(\delta_{N}^{i}, \delta_{N}^{j}\right)$ of the 
weighted pair graph of BCN (2) such that the transition graph of the DFA $A_{\left(\delta_{N}^{i}, \delta_{N}^{j}\right)}$ generated by Algorithm 3.5 has a cycle.

Proof: Since the transition graph has a finite number of vertices, the graph has a cycle iff there is an input sequence $U \in\left(\Delta_{M}\right)^{\mathbb{N}}$ such that $(H L)_{\delta_{N}^{i}}^{\mathbb{N}}(U)=(H L)_{\delta_{N}^{j}}^{\mathbb{N}}(U)$.

In fact, one can determine the observability directly from the weighted pair graph of BCN (2). Theorem 3.14 directly implies the following result.

Theorem 3.15: $\mathrm{BCN}$ (2) is not observable in the sense of Definition 7 iff there is a cycle in its weighted pair graph, and either the cycle contains a non-diagonal vertex, or there is a path from a non-diagonal vertex to the cycle.

Example 3.16: Check whether BCN (3) is observable.

By Theorem 3.15 and Fig. 1, BCN (3) is not observable.

Remark 3.2: An equivalent condition for this observability is given in [14] by checking each pair of distinct periodic state-input trajectories of the same minimal period and same length. In addition, a specific critical length is given in [14] such that if none of the input sequences of that specific length can determine the initial states, nor can input sequences of any other length. Due to the independence of initial states and inputs, their method cannot be used to deal with Definitions 4 or 5 either.

By the end of this subsection, we give a further result on this observability.

Theorem 3.17: Consider BCN (2). Denote the number of non-diagonal vertices of its weighted pair graph by $N_{n d}$. The BCN is observable in the sense of Definition 7, iff $N_{n d}=0$ or, for all distinct states $x_{0}, \bar{x}_{0} \in \Delta_{N}$, for all input sequences $U \in\left(\Delta_{M}\right)^{N_{n d}}, H x_{0}=H \bar{x}_{0}$ implies $(H L)_{x_{0}}^{N_{n d}}(U) \neq(H L)_{\bar{x}_{0}}^{N_{n d}}(U)$.

Proof: "if":

Obvious by Definition 7.

"only if":

Assume that $N_{n d}>0$ and there are distinct $x, x^{\prime} \in \Delta_{N}$ and an input sequence $U \in\left(\Delta_{M}\right)^{N_{n d}}$ such that $H x=H x^{\prime}$ and $(H L)_{x}^{N_{n d}(U)}=(H L)_{x^{\prime}}^{N_{n d}}(U)$. Use Algorithm 3.5 to generate DFA $A_{\left(x, x^{\prime}\right)}=\left(S, \Delta_{M}, \sigma,\left(x, x^{\prime}\right), S\right)$. Then $U \in$ $L\left(A_{\left(x, x^{\prime}\right)}\right)$. Denote $\sigma\left(\left(x, x^{\prime}\right), U\right)$ by $v_{U}$. If $v_{U}$ is diagonal, then $(H L)_{x}^{\mathbb{N}}\left(U\left(\delta_{M}^{1}\right)^{\infty}\right)=(H L)_{x^{\prime}}^{\mathbb{N}}\left(U\left(\delta_{M}^{1}\right)^{\infty}\right)$, and the $\mathrm{BCN}$ is not observable. If $v_{U}$ is not diagonal, there are distinct $i, j \in\left[1, N_{n d}\right]$ such that either $\sigma\left(\left(x, x^{\prime}\right), U[1, i]\right)=$ $\sigma\left(\left(x, x^{\prime}\right), U[1, j]\right)$ or $\left(x, x^{\prime}\right)=\sigma\left(\left(x, x^{\prime}\right), U[1, j]\right)$, for there are exactly $N_{n d}$ non-diagonal vertices. By Theorem 3.15, the $\mathrm{BCN}$ is not observable.

\section{PAirWise NONEQUiVAlENCE OF THE FOUR TYPES OF OBSERVABILITY OF BOOLEAN CONTROL NETWORKS}

In this section, we prove that no pairs of the four types of observability of BCNs are equivalent, which reveals the essence of nonlinearity of BCNs (shown in Fig. 9).

Theorem 4.1: If BCN (2) is observable in the sense of Definition 4, then it is also observable in the sense of Definition 5. The converse is not true.

Proof: The first part naturally follows from Definitions 4 and 5. We use $\mathrm{BCN}$ (3) to prove the second part.
First, we prove that $\mathrm{BCN}$ (3) is not observable in the sense of Definition 4.

Denote $M \quad:=\delta_{4}[1,1,2,1,2,4,1,1] W_{[2,4]} \mathbf{1}_{2}=$ $\delta_{2}[1,2,2,1,1,1,4,1] \mathbf{1}_{2}=\left[\begin{array}{llll}2 & 1 & 0 & 2 \\ 0 & 1 & 1 & 0 \\ 0 & 0 & 0 & 0 \\ 0 & 0 & 1 & 0\end{array}\right]$. Then for all $k \in \mathbb{Z}_{+}$, $M^{k}=\left[\begin{array}{llll}* & * & * & * \\ * & * & * & * \\ 0 & 0 & 0 & 0 \\ 0 & 0 & * & 0\end{array}\right]$. By [7, Theorem 3.3], BCN (3) is not controllable. So one cannot use the test criteria proposed in [6] to check whether BCN (3) is observable.

Next we prove that $\mathrm{BCN}$ (3) is not observable by showing that for state $\delta_{4}^{2}$, there is no input sequence such that the corresponding output sequence can determine it. We only need to consider states $\delta_{4}^{3}, \delta_{4}^{4}$, as $H \delta_{4}^{1} \neq H \delta_{4}^{2}$. Arbitrarily given an input sequence $U \in(\Delta)^{\mathbb{N}}$. If $U(0)=\delta_{2}^{1}$, then $L_{\delta_{4}^{2}}^{1}\left(\delta_{2}^{1}\right)=$ $L_{\delta_{4}^{3}}^{1}\left(\delta_{2}^{1}\right)=\delta_{4}^{2}$. Then for each such $U,(H L)_{\delta_{4}^{2}}^{\mathbb{N}^{4}}(U)=$ $(H L)_{\delta_{4}^{3}}^{\mathbb{N}}(U)$. Else if $U(0)=\delta_{2}^{2}$, then $L_{\delta_{4}^{2}}^{1}\left(\delta_{2}^{2}\right)=L_{\delta_{4}^{4}}^{1}\left(\delta_{2}^{2}\right)=\delta_{4}^{1}$. Then for each such $U,(H L)_{\delta_{4}^{2}}^{\mathbb{N}}(U)=(H L)_{\delta_{4}^{4}}^{\mathbb{N}}(U)$. Then BCN (3) is not observable in the sense of Definition 4.

Second, we prove that BCN (3) is observable in the sense of Definition 5 . We only need to check the state pairs $\left(\delta_{4}^{2}, \delta_{4}^{3}\right)$, $\left(\delta_{4}^{2}, \delta_{4}^{4}\right)$ and $\left(\delta_{4}^{3}, \delta_{4}^{4}\right)$.

For $\left(\delta_{4}^{2}, \delta_{4}^{3}\right),(H L)_{\delta_{4}^{2}}^{1}\left(\delta_{2}^{2}\right)=\delta_{2}^{1} \neq(H L)_{\delta_{4}^{3}}^{1}\left(\delta_{2}^{2}\right)=\delta_{2}^{2}$.

For $\left(\delta_{4}^{2}, \delta_{4}^{4}\right),(H L)_{\delta_{4}^{2}}^{1}\left(\delta_{2}^{1}\right)=\delta_{2}^{2} \neq(H L)_{\delta_{4}^{4}}^{1}\left(\delta_{2}^{1}\right)=\delta_{2}^{1}$.

For $\left(\delta_{4}^{3}, \delta_{4}^{4}\right),(H L)_{\delta_{4}^{3}}^{1}\left(\delta_{2}^{1}\right)=\delta_{2}^{2} \neq(H L)_{\delta_{4}^{4}}^{1}\left(\delta_{2}^{1}\right)=\delta_{2}^{1}$.

Thus, BCN (3) is observable in the sense of Definition 5.

Theorem 4.2: If BCN (2) is observable in the sense of Definition 7, then it is also observable in the sense of Definition 5. The converse is not true.

Proof: The first part follows from Definitions 5 and 7. We also use BCN (3) to prove the second part.

We have proved that $\mathrm{BCN}$ (3) is observable in the sense of Definition 5 in Theorem 4.1. BCN (3) is not observable in the sense of Definition 7, because $H \delta_{4}^{2}=$ $H \delta_{4}^{4}=\delta_{2}[1,2,2,2] \delta_{4}^{2}=\delta_{2}^{2}$ and $(H L)_{\delta_{4}^{2}}^{\mathbb{N}}\left(\delta_{2}^{2}\left(\delta_{2}^{1}\right)^{\infty}\right)=$ $(H L)_{\delta_{4}^{4}}^{\mathbb{N}}\left(\delta_{2}^{2}\left(\delta_{2}^{1}\right)^{\infty}\right)$.

Theorem 4.3: If BCN (2) is observable in the sense of Definition 7, then it is also observable in the sense of Definition 6. The converse is not true.

Proof: Assume that a given BCN (2) is observable in the sense of Definition 7, then arbitrarily given $U \in\left(\Delta_{M}\right)^{\mathbb{N}}$, for any distinct $\delta_{N}^{i}, \delta_{N}^{j}, H \delta_{N}^{i}=H \delta_{N}^{j}$ implies $(H L)_{\delta_{N}^{i}}^{\mathbb{N}}(U) \neq$ $(H L)_{\delta_{N}^{j}}^{\mathbb{N}}(U)$. Since $N<+\infty$, there is $p \in \mathbb{Z}_{+}$such that for any distinct $\delta_{N}^{i}, \delta_{N}^{j}, H \delta_{N}^{i}=H \delta_{N}^{j}$ implies $(H L)_{\delta_{N}^{i}}^{p}(U[0, p-$ 1]) $\neq(H L)_{\delta_{N}^{j}}^{p}(U[0, p-1])$. That is, the BCN is observable in the sense of Definition 6.

To prove the second part, consider the following $\mathrm{BCN}$ :

$$
\begin{aligned}
x(t+1) & =\delta_{4}[1,1,3,3,1,2,3,2] x(t) u(t), \\
y(t) & =\delta_{2}[1,1,2,2] x(t),
\end{aligned}
$$

where $t \in \mathbb{N}, x \in \Delta_{4}, y, u \in \Delta$.

Choose $U=\delta_{2}^{1} \in(\Delta)^{1} . H \delta_{4}^{1}=H \delta_{4}^{2}=\delta_{2}^{1},(H L)_{\delta_{4}^{1}}^{1}(U)=$ $\delta_{2}^{1} \neq(H L)_{\delta_{4}^{2}}^{1}(U)=\delta_{2}^{2} . H \delta_{4}^{3}=H \delta_{4}^{4}=\delta_{2}^{2},(H L)_{\delta_{4}^{3}}^{1^{3}}(U)=$ $\delta_{2}^{1} \neq(H L)_{\delta_{4}^{4}}^{1}(U)=\delta_{2}^{2}$. Then BCN (6) is observable in the sense of Definition 6. 


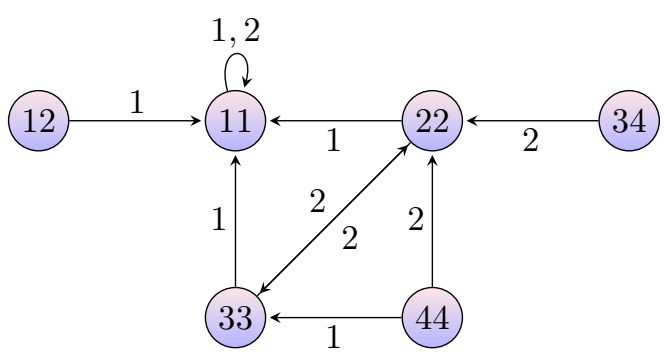

Fig. 6. The weighted pair graph of BCN (7), where the number $i j$ in each circle denotes the state pair $\left(\delta_{4}^{i}, \delta_{4}^{j}\right)$, the weight $k_{1}, k_{2}, \ldots$ beside each edge denotes the weight $\left\{\delta_{2}^{k_{1}}, \delta_{2}^{k_{2}}, \ldots\right\}$ of the edge.

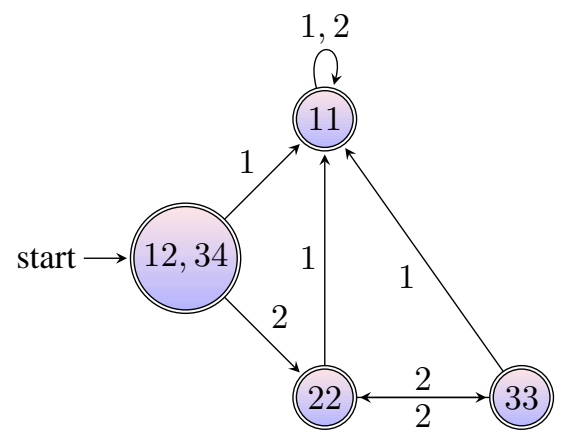

Fig. 7. The DFA $A_{\mathcal{V}_{n}}$ with respect to BCN (7) generated by Algorithm 3.10, where the number $i j$ in each circle denotes the state pair $\left(\delta_{4}^{i}, \delta_{4}^{j}\right)$, the weight $k$ of each edge denotes the input $\delta_{2}^{k}$.

Consider any $U \in(\Delta)^{\mathbb{N}}$ such that $U(0)=\delta_{2}^{2}$. Then $L_{\delta^{3}}^{\mathbb{N}}(U)=L_{\delta_{4}^{4}}^{\mathbb{N}}(U)$ and $(H L)_{\delta_{4}^{3}}^{\mathbb{N}}(U)=(H L)_{\delta_{4}^{4}}^{\mathbb{N}}(U)$. That is, BCN (6) is not observable in the sense of Definition 7.

Theorem 4.4: If BCN (2) is observable in the sense of Definition 6, then it is also observable in the sense of Definition 4. The converse is not true.

Proof: The first part holds naturally. To prove the second part, consider the following $\mathrm{BCN}$ :

$$
\begin{aligned}
x(t+1) & =\delta_{4}[1,1,1,3,1,2,3,2] x(t) u(t), \\
y(t) & =\delta_{2}[1,1,2,2] x(t),
\end{aligned}
$$

where $t \in \mathbb{N}, x \in \Delta_{4}, y, u \in \Delta$.

The weighted pair graph of BCN (7) is as shown in Fig. 6.

The DFA $A_{\left\{\left(\delta_{4}^{1}, \delta_{4}^{2}\right),\left(\delta_{4}^{3}, \delta_{4}^{4}\right)\right\}}$ generated by Algorithm 3.10 (see Fig. 7) is complete. Then by Theorem 3.11, BCN (3) is not observable in the sense of Definition 6.

The DFAs $A_{\delta_{4}^{1}}$ and $A_{\delta_{4}^{3}}$ generated by Algorithm 3.1 (see Fig. 8) satisfy $\delta_{2}^{2} \notin L\left(A_{\delta_{4}^{1}}\right)$ and $\delta_{2}^{1} \notin L\left(A_{\delta_{4}^{3}}\right)$. Then by Theorem 3.2, BCN (7) is observable in the sense of Definition 4.
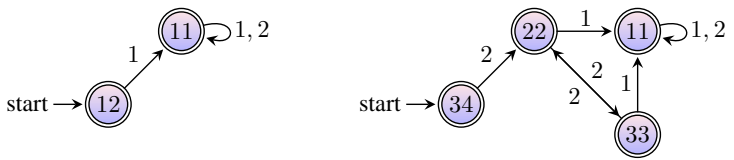

Fig. 8. The DFAs $A_{\delta_{4}^{1}}$ and $A_{\delta_{4}^{3}}$ with respect to $\mathrm{BCN}$ (7) generated by Algorithm 3.1, where the number $i j$ in each circle denotes the state pair $\left(\delta_{4}^{i}, \delta_{4}^{j}\right)$, the weight $k$ beside each edge denotes the input $\delta_{2}^{k}$.

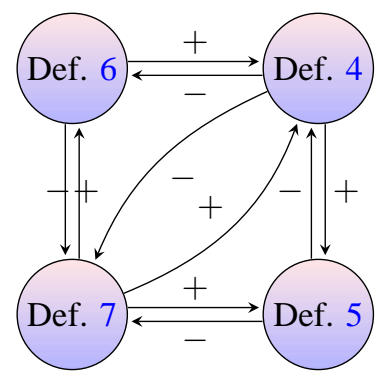

Fig. 9. The implication relationships between Definitions 4, 5, 6 and 7, where "+" means "implies" and "-" means "does not imply".

Theorem 4.5: If BCN (2) is observable in the sense of Definition 7, then it is also observable in the sense of Definition 4. The converse is not true.

Proof: The first part holds naturally. To prove the second part, consider BCN (7) again.

We have proved that $\mathrm{BCN}$ (7) is observable in the sense of Definition 4 in Theorem 4.4. Note that in Fig. 8, the DFAs are just the corresponding ones generated by Algorithm 3.5. Then By Theorem 3.14, BCN (7) is not observable in the sense of Definition 7.

\section{CONCLUDING REMARKS}

In this paper, we solved the problem on determining the observability of Boolean control networks (BCNs) completely by using techniques in finite automata. Also, we showed that no pairs of all the four types of observability notions are equivalent by counterexamples, which reveals the essence of nonlinearity of BCNs (shown in Fig. 9).

Note that the computational complexity of algorithms for determining the first and fourth types of observability is in exponential time, and the algorithms for the other two types are in doubly exponential time. How to reduce the computational complexity effectively is a challenging and urgent problem, and we are naturally concerned with "Is there a nondeterministic polynomial time algorithm for determining the observability of BCNs?" Furthermore, we conjecture that "Determining the observablity of BCNs is PSPACE-hard."

\section{ACKNOWLEDGMENT}

The first author is in debt to Prof. Jarkko Kari, Drs. Charalampos Zinoviadis, Ville Salo and Ilkka Törmä at the University of Turku, Finland for fruitful discussions while visiting the same university in 2013. Both authors thank Mr. Chuang $\mathrm{Xu}$ at the University of Alberta, Canada, the anonymous referees and the associate editor for their valuable comments that highly improve the presentation of this paper.

\section{REFERENCES}

[1] J. Kari. A Lecture Note on Automata and Formal Languages. http://users.utu.fi/jkari/automata/, 2013.

[2] T. Ideker, T. Galitski, L. Hood (2001). A new approach to decoding life: systems biology, Аnnu. Rev. Genomics Hum. Genet., 2, 343-372.

[3] S. A. Kauffman (1969). Metabolic stability and epigenesis in randomly constructed genetic nets, J. Theoretical Biology, 22, 437-467.

[4] D. Cheng (2001). Semi-tensor product of matrices and its application to Morgen's problem, Sci. China Ser. F, 44, 195-212. 
[5] T. Akutsu, M. Hayashida, W. Ching, M. K. Ng (2007). Control of Boolean networks: Hardness results and algorithms for tree structured networks, J. Theoretical Biology, 244, 670-679.

[6] D. Cheng, H. Qi (2009). Controllability and observability of Boolean control networks, Automatica, 45(7), 1659-1667.

[7] Y. Zhao, H. Qi, D. Cheng (2010). Input-state incidence matrix of Boolean control networks and its applications, Systems Control Lett., 59, 767-774.

[8] D. Cheng, Y. Zhao (2011). Identification of Boolean control networks, Automatica, 47, 702-710.

[9] D. Cheng, H. Qi, Z. Li (2011). Analysis and Control of Boolean Networks: A Semi-tensor Product Approach, London: Springer.

[10] L. Zhang, K. Zhang (2013). Controllability and observability of Boolean control networks with time-variant delays in states, IEEE Trans. Neural Networks and Learning Systems, 24, 1478-1484.

[11] R. Li, M. Yang, T. Chu (2013). Observability conditions of Boolean control networks, Int. J. Robust. Nonlinear Control, 24, 2711-2723.

[12] D. Laschov, M. Margaliot, G. Even (2013). Observability of Boolean networks: A graph-theoretic approach, Automatica, 49, 2351-2362.

[13] K. Zhang, L. Zhang, L. Xie (2015). Invertibility and nonsingularity of Boolean control networks, Automatica, 60, 155-164.

[14] E. Fornasini, M. Valcher (2013). Observability, reconstructibility and state observers of Boolean control networks, IEEE Trans. Automat. Control, 58(6), 1390-1401.

[15] X. Xu, Y. Hong (2013). Observability analysis and observer design for finite automata via matrix approach, IET Control Theory and Applications, 7 (12), 1609-1615.

[16] R. Li, M. Yang, T. Chu (2015). Controllability and observability of Boolean networks arising from biology, Chaos: An Interdisciplinary Journal of Nonlinear Science , 25, 023104:1-15. 